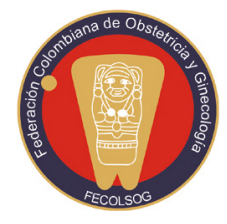

\title{
LA EPISIOTOMÍA EN MUJERES NULÍPARAS: ¿UN HÁBITO DIFÍCIL DE DEJAR?
}

n el presente número de la Revista Colombiana de Obstetricia y Ginecología se publica el estudio de Mellizo-Gaviria et al. titulado "Frecuencia de la realización de episiotomía y complicaciones en el servicio de obstetricia del Hospital Universitario San José, Popayán (Colombia), 2016. Exploración de factores maternos y perinatales asociados a su realización”. Este estudio observacional de corte transversal buscó definir la frecuencia de uso de la episiotomía y sus complicaciones, y explorar algunos factores relacionados con su empleo durante la atención del parto. A partir de un muestreo aleatorio en una institución con una proporción de nacimientos por cesárea reportada en 2015 de $50 \%$, encontraron una frecuencia de $30,5 \%$ en el empleo global de la episiotomía para los nacimientos a término, la cual se realizó en el 58,2\% de las mujeres nulíparas, mientras que solo se llevó a cabo en el 6,6\% de las mujeres multíparas. Como lo mencionan los autores, la nuliparidad incrementó más de ocho veces la probabilidad de realizar una episiotomía en las pacientes atendidas en la institución.

La episiotomía es una intervención concebida para ampliar el introito vaginal y facilitar la salida del feto, lo que podría proteger el piso pélvico, prevenir laceraciones severas del periné, disminuir el riesgo de lesiones hipóxico-isquémicas en el feto y facilitar una restauración anatómica y fisiológica del periné (1). Sin embargo, la evidencia científica no ha demostrado todos los beneficios propuestos, por lo que su empleo rutinario se ha desestimado y, al contrario, las recomendaciones actuales nacionales e internacionales involucran un uso selectivo de esta, aun en presencia de partos instrumentados (2-7).
Como consecuencia de estas recomendaciones puede observarse una tendencia mundial a la disminución de su empleo (8-11), hecho que es más visible en los países de altos ingresos, donde las tasas de episiotomía son inferiores al $30 \%$, mientras que en los países de menores ingresos pueden alcanzarse tasas superiores al $70 \%$ (11).

En Colombia, las tasas de episiotomía han tenido una tendencia decreciente según diferentes reportes de la literatura, y han pasado de emplearse en más del $50 \%$ de todos los nacimientos, hasta tasas entre 12 y el $30 \%$ en los últimos veinte años (12). No obstante, la reducción en las tasas de utilización de la episiotomía en mujeres nulíparas no es tan evidente. Mientras entre 2001 y 2005 se reportaron tasas de empleo en nulíparas cercanas al $90 \%(9,12)$, reportes recientes, incluido el artículo publicado en el presente número, reportan su empleo entre el 60 y el $70 \%$ de estas mujeres. En Colombia, en algunos hospitales, esta frecuencia ha disminuido por debajo del $30 \%$ mediante políticas estrictas de episiotomía selectiva (12, 13) que acercan la tasa global de este procedimiento al 10\% recomendado por la Organización Mundial de la Salud (OMS) desde 1996 (14).

El empleo y la indicación de la episiotomía dependen del criterio y la experiencia de quien atiende a la gestante, dado que con la evidencia disponible no hay consenso sobre los estándares e indicaciones que orienten la práctica clínica $(7,8)$. El énfasis en el empleo de la episiotomía en las mujeres nulíparas debe llamar la atención sobre las preferencias del obstetra, su formación y el papel que desempeña en la formación del talento humano en salud en los hos- 
pitales universitarios, pues es su deber supervisar el personal en entrenamiento para enseñarle a reconocer aquellas pacientes con riesgo de desgarro perineal severo a fin de ofrecer oportunamente medidas efectivas para la prevención de estos eventos, como compresas tibias, enseñar las técnicas de protección del periné e indicar selectivamente la episiotomía (4, 5, 12, 13).

El manejo restrictivo de la episiotomía es primordial, así como incentivar la disponibilidad de métodos para el manejo del dolor, la ambulación durante el trabajo de parto, permitir el acompañamiento permanente, la ingesta de líquidos y la adopción libre de la posición materna al momento del parto $(6,7$, 14-16), a fin de favorecer los resultados maternos y perinatales, humanizar la experiencia para todas las personas que participan en el nacimiento, y prevenir la percepción de violencia obstétrica por parte de las mujeres y sus familias.

\section{Jorge Andrés Rubio-Romero MD, MSc, FACOG}

Profesor Titular

Departamento de Obstetricia y Ginecología Universidad Nacional de Colombia

Editor Asociado

Revista Colombiana de Obstetricia y Ginecología

\section{REFERENCIAS}

1. Rubio-Romero JA. Indicaciones actuales de la episiotomía. Rev Fac Med. 2004;52(2):132-9.

2. Jiang H, Qian X, Carroli G, Garner P. Selective versus routine use of episiotomy for vaginal birth. Cochrane Database Syst Rev. 2017;2:CD000081. https://doi. org/10.1002/14651858.CD000081.pub3

3. Guidelines for operative vaginal birth. J Obstet Gynaecol Can. 2004;26(8):747-53. https://doi. org/10.1016/S1701-2163(16)30647-8

4. Aasheim V, Nilsen ABV, Lukasse M, Reinar LM. Perineal techniques during the second stage of labour for reducing perineal trauma. En: The Cochrane Collaboration, editor. Cochrane Database of Systematic Reviews. Chichester, UK: John Wiley \& Sons; 2011 (visitado 2018 junio 26). https://doi.wiley. com/10.1002/14651858.CD006672.pub2

5. American College of Obstetricians and Gynecologists' Committee on Practice Bulletins-Obstetrics. Practice Bulletin 165: Prevention and management of obstetric lacerations at vaginal delivery. Obstet Gynecol. 2016;128(1):e1-15. https://doi.org/10.1097/ AOG.0000000000001523

6. Colombia. Ministerio de Salud y Protección Social. Guía de Práctica Clínica (GPC) para la prevención, detección temprana y tratamiento de las complicaciones del embarazo, parto o puerperio (visitado 2018 junio 26). Disponible en: http://gpc.minsalud.gov.co/ gPc_sites/Repositorio/Conv_500/GPC_embarazo/ gpc_embarazo.aspx

7. WHO | Managing complications in pregnancy and childbirth: A guide for midwives and doctors, $2 \mathrm{ed}$ WHO. (visitado 2018 junio 26). Disponible en: http:// www.who.int/maternal_child_adolescent/documents/ managing-complications-pregnancy-childbirth/en/

8. Hale RW, Ling FW. Episiotomy: Procedure and repair techniques. Washington, D.C: American College of Obstetricians and Gynecologists; 2007. p. 24.

9. Graham ID, Carroli G, Davies C, Medves JM. Episiotomy rates around the world: an update. Birth Berkeley Calif. 2005;32(3):219-23. https://doi. org/10.1111/j.0730-7659.2005.00373.x

10. Conde-Agudelo A, Rosas-Bermudez A, Gülmezoglu AM. Evidence-based intrapartum care in Cali, Colombia: A quantitative and qualitative study. BJOG Int J Obstet Gynaecol. 2008;115(12):1547-56. https:// doi.org/10.1111/j.1471-0528.2008.01930.x

11. Clesse C, Lighezzolo-Alnot J, De Lavergne S, Hamlin S, Scheffler M. Statistical trends of episiotomy around the world: Comparative systematic review of changing practices. Health Care Women Int. 2018;39(6):644-62.

12. Rubio JA. Política selectiva de episiotomía y riesgo de desgarro perineal en un hospital universitario. Rev Colomb Obstet Ginecol. 2005;56(2):116-26.

13. Abril-González FP, Guevara-Villareal AS, Ramos-Cruz A, Rubio-Romero JA. Risk factors for perineal tearing 
during births without episiotomy attended by personnel being trained at a teaching hospital in Bogotá, Colombia 2007. Rev Colomb Obstet Ginecol. 2009;60(2):143-51.

14. Technical Working Group, World Health Organization. Care in normal birth: A practical guide. Birth Berkeley. 1997;24(2):121-3. https://doi.org/10.1111/j.1523536X.1997.tb00352.x

15. Rubio-Romero JA, Ruiz-Parra AI, Martínez F, MuñozRestrepo J, Muñoz LA, Arévalo-Rodríguez I, et al. Guía de Práctica Clínica para la detección temprana de las anomalías durante el trabajo de parto, atención del parto normal y distócico. Rev Colomb Obstet Ginecol. 2013;64(4):379-424.

16. OMS | Recomendaciones de la OMS para la conducción del trabajo de parto. WHO (visitado 2018 jun 26). Disponible en: http://www.who.int/reproductivehealth/ publications/maternal_perinatal_health/augmentationlabour/es/ 